\title{
Hedgehog signaling, epithelial-to-mesenchymal transition and miRNA (Review)
}

\author{
YURIKO KATOH ${ }^{1}$ and MASARU KATOH ${ }^{2}$ \\ ${ }^{1}$ M\&M Medical Bioinformatics, Hongo 113-0033; ${ }^{2}$ Genetics and \\ Cell Biology Section, National Cancer Center, Tokyo 104-0045, Japan
}

Received May 19, 2008; Accepted June 26, 2008

DOI: 10.3892/ijmm_00000019

\begin{abstract}
SHH, IHH, and DHH are lipid-modified secreted proteins binding to Patched receptors, and CDON, BOC or GAS1 co-receptors. In the absence of Hedgehog signaling, GLI1 is transcriptionally repressed, GLI2 is phosphorylated by GSK3 and CK1 for the FBXW11 (ßTRCP2)-mediated degradation, and GLI3 is processed to a cleaved repressor. In the presence of Hedgehog signaling, Smoothened is relieved from Patched-mediated suppression due to the Hedgehogdependent internalization of Patched, which leads to MAP3K10 (MST) activation and SUFU inactivation for the stabilization and nuclear accumulation of GLI family members. GLI activators then upregulate $C C N D 1, C C N D 2$ for cell cycle acceleration, FOXA2, FOXC2, FOXE1, FOXF1, FOXL1, FOXP3, POU3F1, RUNX2, SOX13, TBX2 for cell fate determination, $J A G 2, I N H B C$, and INHBE for stem cell signaling regulation. Hedgehog signals also upregulate SFRP1 in mesenchymal cells for WNT signaling regulation. Epithelial-to-mesenchymal transition (EMT) during embryogenesis, adult tissue homeostasis and carcinogenesis is characterized by class switch from E-cadherin to N-cadherin. SNAI1 (Snail), SNAI2 (Slug), SNAI3, ZEB1, ZEB2 (SIP1), KLF8, TWIST1, and TWIST2 are EMT regulators repressing $\mathrm{CDHl}$ gene encoding E-cadherin. Hedgehog signals induce JAG2 upregulation for Notch-CSL-mediated SNAI1 upregulation, and also induce TGFß1 secretion for ZEB1 and ZEB2 upregulation via TGFß receptor and NF-кB. TGFßmediated downregulation of miR-141, miR-200a, miR-200b, miR-200c, miR-205, and miR-429 results in upregulation of ZEB1 and ZEB2 proteins. Hedgehog signaling activation indirectly leads to EMT through FGF, Notch, TGFß signaling cascades, and miRNA regulatory networks. miRNAs targeted to stem cell signaling components or EMT regulators are potent drug targets; however, off-target effects should be
\end{abstract}

Correspondence to: Dr Masaru Katoh, Genetics and Cell Biology Section, National Cancer Center, 5-1-1 Tsukiji, Chuo Ward, Tokyo 104-0045, Japan

E-mail: mkatoh-kkr@umin.ac.jp

Key words: Hedgehog, epithelial-mesenchymal transition, Ecadherin, N-cadherin, Snail, Slug, ZEB1, ZEB2, Twist, miRNA strictly controlled before clinical application of synthetic miRNA. Peptide mimetic and RNA aptamer could also be utilized as Hedgehog signaling inhibitors or EMT suppressors.

\section{Contents}

1. Introduction

2. Hedgehog signaling pathway

3. Epithelial-to-mesenchymal transition (EMT)

4. Hedgehog and EMT

5. MicroRNA (miRNA)

6. Perspectives

\section{Introduction}

Hedgehog family members are key regulators of embryogenesis, adult tissue homeostasis, and carcinogenesis (1-5). Genetic alterations and aberrant expression of Hedgehog signaling molecules in medulloblastoma, glioma, basal cell carcinoma, lung cancer, esophageal cancer, gastric cancer, pancreatic cancer, prostate cancer, and ovarian cancer have been reported, and reviewed elsewhere.

Hedgehog signaling cascade cross talks with WNT, Notch, EGF/FGF, and TGFß/Activin/Nodal/BMP signaling cascades to constitute the stem cell signaling network (6-9). Deregulation of the stem cell signaling network due to the accumulation of germline mutation, SNP, chronic inflammation, epigenetic change, and genetic alteration leads to carcinogenesis (10).

Epithelial cells undergo fibroblastoid morphological changes associated with increased motility or invasiveness due to decreased cell-cell adhesion (11-14). Fibroblastoid morphological changes of epithelial cells are known as epithelial-to-mesenchymal transition (EMT).

Regulatory mechanisms of EMT are hot issues in life science, especially in the fields of developmental biology and oncology. Herein recent advances in the Hedgehog research will be reviewed with the emphasis on EMT and microRNA (miRNA).

\section{Hedgehog signaling pathway}

Sonic Hedgehog (SHH), Indian Hedgehog (IHH), and Desert Hedgehog (DHH) are mammalian Hedgehog family ligands, 

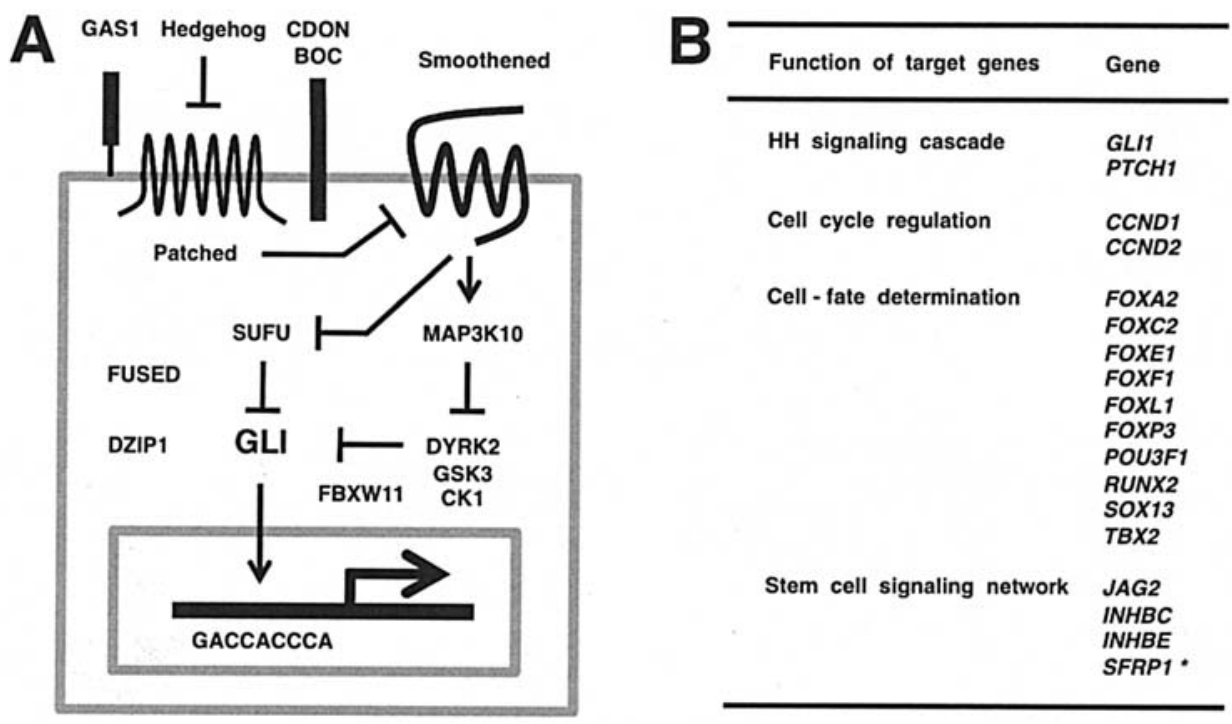

Figure 1. Hedgehog signaling pathway. (A) Schematic representation of Hedgehog signaling cascade. Patched family members are Hedgehog receptors, while CDON, BOC and GAS1 are Hedgehog co-receptors. In the absence of Hedgehog signaling, GLI1 is transcriptionally repressed, GLI2 is phosphorylated by GSK3 and CK1 for the FBXW11-mediated degradation, and GLI3 is processed to a cleaved repressor. In the presence of Hedgehog signaling, Smoothened is relieved from Patched-mediated suppression to induce MAP3K10 activation and SUFU inactivation. GLI activators then bind to the GACCACCCA motif for the transcriptional upregulation of target genes. (B) List of representative Hedgehog target genes.

consisting of N-terminal signal peptide, Hedgehog core domain, and C-terminal processing domain (15-17). Hedgehog precursors are autoprocessed to cut off the C-terminal processing domain for cholesteroylation, and then further processed by Hedgehog acyltransferase (HHAT) to cut off the N-terminal signal peptide for palmitoylation $(18,19)$. Mature Hedgehog proteins with lipid modifications are then transported to the cell surface for packaging into lipoprotein particles depending on Dipatched 1 (DISP1), or for multimerization via lipophilic tails $(19,20)$. Mature Hedgehog proteins secreted from producing cells induce concentrationdependent effects on target cells expressing Hedgehog receptors.

Patched family members, PTCH1 and PTCH2, are Hedgehog receptors distantly related to Dispatch family members with multi-transmembrane domains and a sterolsensing domain $(21,22)$. PTCH1 and PTCH2 do not directly transduce Hedgehog signals to the intracellular signaling cascade, but indirectly through the Smoothened seventransmembrane-type receptor (23). Patched family members, inhibiting Smoothened function, are rapidly internalized upon Hedgehog-binding (24). Due to the release of Smoothened from Patched-dependent suppression, Hedgehog-binding to Patched family receptors indirectly activates the SmoothenedGLI signaling cascade (Fig. 1A).

$\mathrm{CDON}$ and BOC are transmembrane proteins with extracellular immunoglobulin-like (Ig-like) and fibronectin type III (FNIII) domains, which enhance Hedgehog signaling activity as co-receptors (25). GAS1 is a GPI-anchored cell surface protein binding to Hedgehog ligands for the potentiation of Hedgehog signaling (26). On the other hand, HHIP1/HHIP is a Hedgehog-bindng protein to compete Patched receptors for Hedgehog-binding $(27,28)$.

GLII gene was initially cloned as an oncogene amplified in malignant glioma, and then characterized as a transcription factor functioning as a Hedgehog signaling effector $(29,30)$.
GLI1, GLI2, and GLI3 are human homologs of Drosophila Cubitus interruptus. In the absence of Hedgehog signaling, GLI1 is transcriptionally repressed, GLI2 is phosphorylated by GSK3 and CK1 for the FBXW11 (BTRCP2)-mediated degradation, and GLI3 is processed to a cleaved repressor $(30,31)$. In the presence of Hedgehog signaling, Smoothened induces MAP3K10 (MST) activation and SUFU inactivation for the stabilization and nuclear accumulation of GLI family members, respectively $(32,33)$. GLI1 functions as transcriptional activator of Hedgehog target genes, while GLI2 and GLI3 as transcriptional activator or repressor in a context-dependent manner (30).

Hedgehog signaling activation leads to transcriptional activation of target genes through GLI-binding to the GACC ACCCA motif (34-36). GLI1, PTCH1 and HHIP1 are upregulated by Hedgehog signaling, but $C D O N, B O C$ and GAS1 are downregulated. Hedgehog-dependent GLII upregulation constitutes a positive feedback loop, while Hedgehogdependent regulation of PTCH1, HHIP1,CDON, BOC, and GAS1 constitutes a negative feedback network. Hedgehog signals induce transient upregulation of target genes through the combination of positive and negative feedback mechanisms.

Hedgehog signals upregulate $C C N D 1$ and $C C N D 2$ for cell cycle acceleration, FOXA2, FOXC2, FOXE1, FOXF1, FOXL1, FOXP3, POU3F1, RUNX2, SOX13, and TBX2 for cell fate determination. Hedgehog signals also upregulate $J A G 2$, and $I N H B C / E$ to regulate Notch, and Activin signaling cascades, respectively. In addition, Hedgehog signals upregulate $S F R P 1$ at least in mesenchymal cells without its promoter $\mathrm{CpG}$ hypermethylation to inhibit canonical WNT signaling cascade in epithelial cells (Fig. 1B).

\section{Epithelial-to-mesenchymal transition (EMT)}

Epithelial cells are tightly held together with uniform neighboring cells to move as a sheet en block, while mesen- 


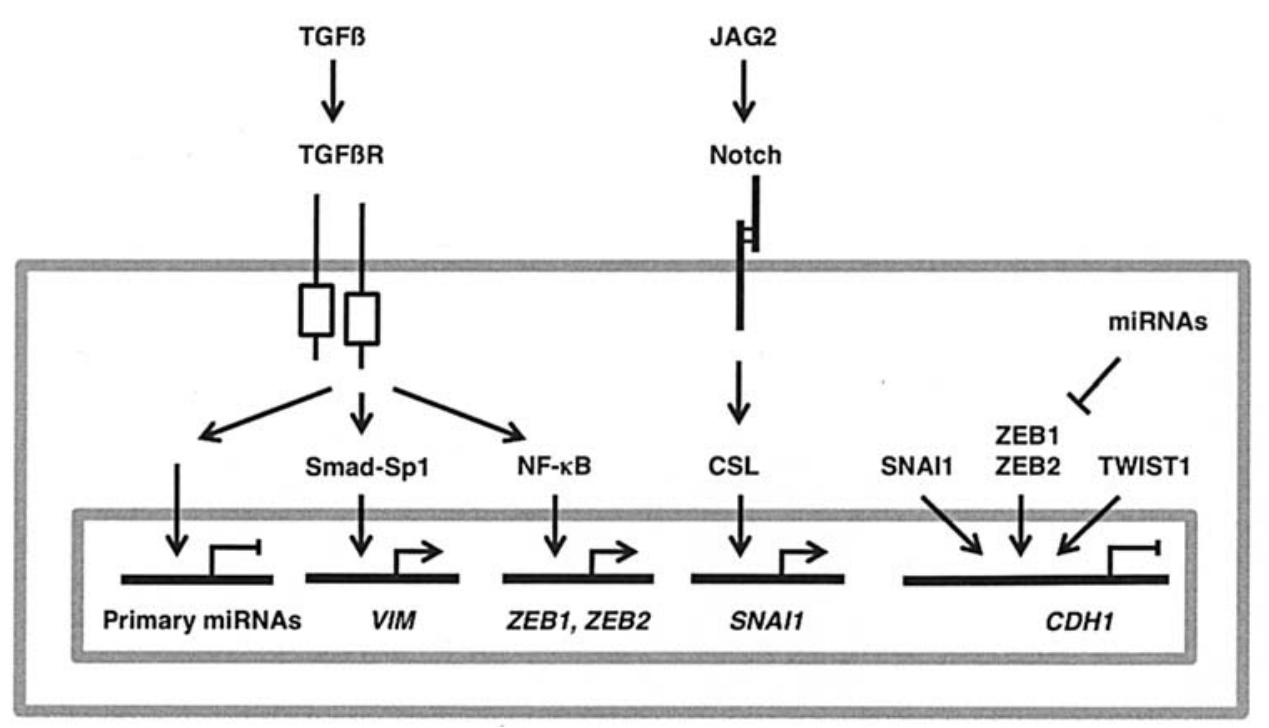

Figure 2. Hedgehog and EMT. Hedgehog signals induce JAG2 upregulation for Notch-CSL-mediated SNAII upregulation, and also induce TGFß1 secretion for ZEB1 and ZEB2 upregulation via TGFß receptor and NF-кB. TGFß-mediated downregulation of miR-141, miR-200a, miR-200b, miR-200c, miR-205, and miR-429 results in upregulation of ZEB1 and ZEB2 proteins. Hedgehog signaling activation indirectly leads to EMT through Notch, TGFß signaling cascades, and miRNA regulatory networks.

chymal cells are loosely connected with diverse neighboring cells to move individually. EMT, allowing cells to dissociate from epithelial tissue, is necessary for gastrulation movements and neural crest formation during embryogenesis, and also for invasion and metastasis during carcinogenesis (11-14).

E-cadherin, occludin and cytokeratin are downregulated during EMT, while N-cadherin, vimentin, fibronectin, SNAI1/SAIL, SNAI2/SLUG, ZEB2/SIP1, and TWIST1 are upregulated (14). E-cadherin and N-cadherin are representative adhesion molecules expressed on epithelial cells and mesenchymal cells, respectively. E-cadherin at the adherens junction is implicated in the stable cell-cell contact of epithelial cells, while $\mathrm{N}$-cadherin in the weak intercellular contact of mesenchymal cells $(37,38)$. Class switch from E-cadherin to $\mathrm{N}$-cadherin results in the loss of epithelial phenotype and the acquisition of mesenchymal phenotype. Transcriptional repression of $C D H 1$ gene or functional repression of E-cadherin protein is the critical step for EMT.

Zinc-finger domain proteins SNAI1, SNAI2, SNAI3, ZEB1, ZEB2, KLF8 as well as basic helix-loop-helix (bHLH) domain proteins TWIST1 and TWIST2, bind to the proximal promoter region of the $\mathrm{CDHl}$ gene for the EMT induction through E-cadherin repression (11-14,39-42). We previously reported SNAII expression in neuroblastoma, diffuse type gastric cancer, and SNAI2 expression in embryonic stem cells, leiomyosarcoma, neuroblastoma, and glioblastoma (41). Rosivatz et al reported preferential upregulation of ZEB2 in intestinal type gastric cancer, and those of SNAI1 and TWIST1 in diffuse type gastric cancer (39). Alves et al reported co-expression of ZEB2 and SNAI2 in intestinal type gastric cancer, and that of SNAII and SNAI2 in diffuse type gastric cancer (42). Upregulation of EMT regulators is associated with more malignant phenotypes in a variety of human cancer, such as gastric cancer, pancreatic cancer, breast cancer, and ovarian cancer.

\section{Hedgehog and EMT}

Hedgehog signaling cascade cross-talks with WNT, EGF/ FGF, and TGFß/Activin/Nodal/BMP signaling cascades, which are implicated in EMT through E-cadherin repression (6-14). In this section, direct and indirect mechanisms of EMT regulation by the Hedgehog signaling cascade will be reviewed (Fig. 2).

Upregulation of SNAII and PTCHI mRNAs is induced $3 \mathrm{~h}$ after GLI1 expression in RK3E cells by using the 'tet-on' system, and that of Snai1 protein $12 \mathrm{~h}$ after GLI1 expression (43). Although these facts indicate that the Hedgehog signaling cascade induces the SNAI1 upregulation, there is no evidence for the direct transcriptional activation of SNAII by the Hedgehog signaling cascade.

On the other hand, Hedgehog signals induce JAG2 upregulation (Fig. 1B), and TGFß1 secretion to promote motility and invasiveness of cancer cells (44). JAG2 signal induces processing of Notch receptor to Notch intracellular domain (NICD). NICD is then associated with CSL transcription factor in the nucleus to induce $S N A I 1$ upregulation (45). TGFß1 signal activates TGFß receptor for the NF-кBmediated transcriptional upregulation of ZEB1 and ZEB2 (46), and also for the SMAD-Sp1-mediated transcriptional upregulation of mesenchymal markers, such as Vimentin (VIM). Together these facts indicate that the Hedgehog signals indirectly induce EMT through the upregulation of multiple EMT regulators via Notch and TGFß signaling cascades (Fig. 2).

\section{MicroRNA (miRNA)}

Primary miRNAs are processed by Drosha/DGCR8 complex to give rise to precursor miRNAs, which are then processed by Dicer to produce mature miRNAs. Most target mRNAs with partial complementarity to miRNA are repressed 
through translational downregulation and deadenylation, while several target mRNAs are activated (47-50). Mechanisms of miRNA-induced translational or transcriptional regulation as well as clinical application of miRNA are the frontier of medical science in the post-genome era.

Zebrafish miR-214 binds to the 3'-UTR of Sufu to downregulate Sufu (51). Because Sufu is implicated in the nuclear trafficking of Gli activator and repressor, miR-214-induced downregulation of Sufu results in maximal activation of Gli in the presence of Hedgehog and complete repression of in the absence of Hedgehog. It is noteworthy that human miR214, up-regulated in ovarian cancer, binds to the 3'-UTR of PTEN to downregulate PTEN for the activation of PI3K-AKT signaling cascade (52), which is also able to induce EMT. Effects of human ortholog of zebrafish miR-214 on the Hedhehog-GLI signaling cascade as well as EMT remain to be elucidated.

TGFß downregulates the expression of human miR-141, miR-200a, miR-200b, miR-200c, miR-205, and miR-429, which are targeted to ZEB1 and ZEB2 mRNAs (53). TGFßinduced downregulation of miRNAs mentioned above synergizes with $\mathrm{NF}-\kappa \mathrm{B}$ sinaling to induce upregulation of ZEB1 and ZEB2 (Fig. 2).

SNAI, ZEB and TWIST family members repress the $C D H 1$ gene to induce EMT, but also regulate the transcription of other target genes. TWIST1 is upregulated in human breast cancer, gastric cancer, esophageal cancer, and prostate cancer. TWIST1 directly activates the transcription of miR-10b primary miRNA, located within the HOXD gene cluster (54). HOXD10 mRNA is the target of miR-10b in human breast cancer, and HOXD10 is implicated in the suppression of RhoC-mediated cell motility. TWIST1 promotes invasion and metastasis through miR-10b-induced HOXD10 repression.

\section{Perspectives}

KAAD-cyclopamine, SANT1-4, and Cur61414 are smallmolecule Hedgehog signaling inhibitors targeting Smoothened (55). Other therapeutic devices targeted to the Hedgehog signaling cascade and EMT regulators will be described in the last section.

miRNAs targeted to mRNAs, encoding stem cell signaling components or EMT regulators, are potent drug targets. miRNAs inducing proliferative, anti-apoptotic, pro-angiogenic, or pro-metastatic effects on tumor cells could be downregulated for cancer therapy, while those inducing proapoptotic, anti-angiogenic, or anti-metastatic effects could be applied for synthetic miRNA $(48,49)$. Because the off-target effects are a serious problem associated with miRNA and siRNA technologies, great care should be taken before clinical application of these technologies.

RNA aptamer is a short RNA oligonucleotide with a stable three-dimensional structure (49). RNA aptamers binding to extracellular region of PTCH1 could be utilized for drug delivery to cancer cells with Hedgehog signaling activation. RNA apatamers binding to cytoplasmic region of SMO, and those binding to Fused or GLI1 could be utilized as Hedgehog signaling inhibitors.

Peptide mimetics, resembling WNT and FGF family members, have been developed $(56,57)$. Because WNT5A transduces signals through ROR1 or ROR2 to activate the non-canonical signaling cascade for the induction of EMT partly through SNAI1 upregulation (58-61), WNT5A mimetic is able to suppress invasion and metastasis of cancer cells. Peptide mimetic, resembling core region of mature Hedgehog signaling domain, could be developed as a novel Hedgehog antagonist.

\section{References}

1. Beachy PA, Karhadkar SS and Berman DM: Tissue repair and stem cell renewal in carcinogenesis. Nature 432: 324-331, 2004.

2. Hooper JF and Scott MP: Communicating with Hedgehogs. Nature Rev Mol Cell Biol 6: 306-317, 2005.

3. Berman DM, Karhadkar SS, Maitra A, et al: Widespread requirement for Hedgehog ligand stimulation in growth of digestive tract tumors. Nature 425: 846-851, 2003.

4. Katoh Y and Katoh M: Hedgehog signaling in gastric cancer. Cancer Biol Ther 4: 1050-1054, 2005.

5. Katoh $\mathrm{Y}$ and Katoh M: Hedgehog signaling pathway and gastrointestinal stem cell signaling network. Int J Mol Med 18: 1019-1023, 2006.

6. Van den Brink GR, Bleuming SA, Hardwick JC, et al: Indian Hedgehog is an antagonist of Wnt signaling in colonic epithelial cell differentiation. Nat Genet 36: 277-282, 2004.

7. Garciadiego-Cazares D, Rosales C, Katoh M and ChimalMonroy J: Coordination of chondrocyte differentiation and joint formation by $\alpha 5 \beta 1$ integrin in the developing appendicular skeleton. Development 131: 4735-4742, 2004.

8. Bailey J, Singh PK and Hollingsworth MA: Cancer metastasis facilitated by developmental pathways: Sonic hedgehog, Notch, and bone morphogenetic proteins. J Cell Biochem 102: 829-839, 2007.

9. Katoh M: Networking of WNT, FGF, Notch, BMP, and Hedgehog signaling pathways during carcinogenesis. Stem Cell Rev 3: 30-38, 2007.

10. Katoh M: Dysregulation of stem cell signaling network due to germline mutation, SNP, Helicobacter pylori infection, epigenetic change, and genetic alteration in gastric cancer. Cancer Biol Ther 6: 832-839, 2007.

11. Thiery JP: Epithelial-mesenchymal transitions in tumour progression. Nature Rev Cancer 2: 442-454, 2002.

12. Barrallo-Gimeno A and Nieto MA: The Snail genes as inducers of cell movement and survival: implications in development and cancer. Development 132: 3151-3161, 2005.

13. Katoh M: Epithelial-mesenchymal transition in gastric cancer. Int J Oncol 27: 1677-1683, 2005.

14. Lee JM, Dedhar S, Kalluri R and Thompson EW: The epithelial-mesenchymal transition: new insights in signaling, development, and disease. J Cell Biol 172: 973-981, 2006.

15. Marigo V, Roberts DJ, Lee SM, et al: Cloning, expression, and chromosomal location of $\mathrm{SHH}$ and $\mathrm{IHH}$. Genomics 28: 44-51, 1995

16. Katoh Y and Katoh M: Identification and characterization of rat Desert hedgehog and Indian hedgehog genes in silico. Int $\mathbf{J}$ Oncol 26: 545-549, 2005.

17. Katoh Y and Katoh M: Comparative genomics on Sonic hedgehog orthologs. Oncol Rep 14: 1087-1090, 2005.

18. Chamoun Z, Mann RK, Nellen D, et al: Skinny hedgehog, an acyltransferase required for palmitoylation and activity of the hedgehog signal. Science 293: 2080-2084, 2001.

19. Breitling R: Greased hedgehogs: new links between hedgehog signaling and cholesterol metabolism. Bioessays 29: 1085-1094, 2007.

20. Burke R, Nellen D, Bellotto M, et al: Dispatched, a novel sterolsensing domain protein dedicated to the release of cholesterolmodified hedgehog from signaling cells. Cell 99: 803-815, 1999.

21. Johnson RL, Rothman AL, Xie J, et al: Human homolog of patched, a candidate gene for the basal cell nevus syndrome. Science 272: 1668-1671, 1996.

22. Katoh Y and Katoh M: Identification and characterization of DISP3 gene in silico. Int J Oncol 26: 551-556, 2005.

23. Van den Heuvel M and Ingham PW: Smoothened encodes a receptor-like serpentine protein required for hedgehog signalling. Nature 382: 547-551, 1996. 
24. Gallet A and Therond PP: Temporal modulation of the Hedgehog morphogen gradient by a Patched-dependent targeting to lysosomal compartment. Dev Biol 277: 51-62, 2005.

25. Tenzen T, Allen BL, Cole F, et al: The cell surface membrane proteins $\mathrm{Cdo}$ and Boc are components and targets of the Hedgehog signaling pathway and feedback network in mice. Dev Cell 10: 647-656, 2006.

26. Allen BL, Tenzen T and McMahon AP: The Hedgehog-binding proteins Gas 1 and Cdo cooperate to positively regulate Shh signaling during mouse development. Genes Dev 21: 1244-1257, 2007.

27. Chuang PT and McMahon AP: Vertebrate Hedgehog signalling modulated by induction of a Hedgehog-binding protein. Nature 397: 617-621, 1999.

28. Katoh Y and Katoh M: Comparative genomics on HHIP family orthologs. Int J Mol Med 17: 391-395, 2006.

29. Kinzler KW, Bigner SH, Bigner DD, et al: Identification of an amplified, highly expressed gene in a human glioma. Science 236: 70-73, 1987.

30. Ruiz i Altaba A, Mas C and Stecca B: The Gli code: an information nexus regulating cell fate, stemness and cancer. Trends Cell Biol 17: 438-447, 2007.

31. Bhatia N, Thiyagarajan S, Elcheva I, et al: Gli2 is targeted for ubiquitination and degradation by $ß$-TrCP ubiquitin ligase. J Biol Chem 281: 19320-19326, 2006.

32. Katoh M, Hirai M, Sugimura T and Terada M: Cloning and characterization of MST, a novel serine/threonine kinase with SH3 domain. Oncogene 10: 1447-1451, 1995.

33. Varjosalo M, Björklund M, Cheng F, et al: Application of active and kinase-deficient kinome collection for identification of kinases regulating hedgehog signaling. Cell 133: 537-548, 2008.

34. Hallikas O, Palin K, Sinjushina N, et al: Genome-wide prediction of mammalian enhancers based on analysis of transcription-factor binding affinity. Cell 124: 47-59, 2006.

35. Kasper M, Schnidar H, Neill GW, et al: Selective modulation of Hedgehog/GLI target gene expression by EGF signaling in human keratinocytes. Mol Cell Biol 26: 6283-6299, 2006.

36. Katoh Y and Katoh M: WNT antagonist, SFRP1, is Hedgehog signaling target. Int J Mol Med 17: 171-175, 2006.

37. Takeichi M: The cadherins: cell-cell adhesion molecules controlling animal morphogenesis. Development 102: 639-655, 1988.

38. Hazan RB, Phillips GR, Qiao RF, et al: Exogenous expression of $\mathrm{N}$-cadherin in breast cancer cells induces cell migration, invasion and metastasis. J Cell Biol 148: 779-790, 2000

39. Rosivatz E, Becker I, Specht K, et al: Differential expression of the EMT regulators Snail, SIP1, and Twist in gastric cancer. Am J Pathol 161: 1881-1891, 2002.

40. Katoh M and Katoh M: Identification and characterization of human SNAIL3 (SNAI3) gene in silico. Int J Mol Med 11: 383-388, 2003

41. Katoh $\mathrm{M}$ and Katoh M: Comparative genomics on SNAI1, SNAI2, and SNAI3 orthologs. Oncol Rep 14: 1083-1086, 2005.

42. Alves CC, Rosivatz E, Schott C, et al: Slug is overexpressed in gastric carcinomas and may act synergistically with SIP1 and Snail in the down-regulation of E-cadherin. J Pathol 211: 507-515, 2007.

43. Li X, Deng W, Nail CD, et al: Snail induction is an early response to Gli1 that determines the efficiency of epithelial transformation. Oncogene 25: 609-621, 2006.
44. Yoo YA, Kang MH, Kim JS and Oh SC: SHH signaling promotes motility and invasiveness of gastric cancer cells through TGF3-mediated activation of the ALK5-Smad3 pathway. Carcinogenesis 29: 480-490, 2008.

45. Sahlgren C, Gustafsson MV, Jin S, Poellinger L and Lendahl U: Notch signaling mediates hypoxia-induced tumor cell migration and invasion. Proc Natl Acad Sci USA 105: 6392-6397, 2008.

46. Chua HL, Bhat-Nakshatri $\mathrm{P}$, Clare $\mathrm{SE}$, et al: $\mathrm{NF}-\kappa \mathrm{B}$ represses E-cadherin expression and enhances EMT of mammary epithelial cells: potential involvement of ZEB1 and ZEB2. Oncogene 26: 711-724, 2007

47. Vasudevan S, Tong Y and Steitz JA: Switching from repression to activation: microRNAs can up-regulate translation. Science 318: 1931-1934, 2007.

48. Marquez RT and McCaffrey AP: Advances in microRNAs: implication for gene therapists. Hum Gene Ther 19: 27-38, 2008

49. Katoh M: RNA technology targeted to the WNT signaling pathway. Cancer Biol Ther 7: 275-277, 2008

50. Grosshans $\mathrm{H}$ and Filipowicz W: The expanding world of small RNAs. Nature 451: 414-416, 2008.

51. Flynt AS, Li N, Thatcher EJ, Solnica-Krezel L and Patton JG: Zebrafish miR-214 modulates Hedgehog signaling to specify muscle cell fate. Nat Genet 39: 259-263, 2007.

52. Yang H, Kong W, He L, et al: MicroRNA expression profiling in human ovarian cancer: miR-214 induces cell survival and cisplatin resistance by targeting PTEN. Cancer Res 68: 425-433 2008.

53. Gregory PA, Bert AG, Paterson EL, et al: The miR-200 family and miR-205 regulate epithelial to mesenchymal transition by targeting ZEB1 and SIP1. Nat Cell Biol 10: 593-601, 2008.

54. Ma L, Teruya-Feldstein J and Weinberg RA: Tumour invasion and metastasis initiated by microRNA-10b in breast cancer. Nature 449: 682-689, 2007.

55. Williams JA, Guicherit OM, Zaharian BI, et al: Identification of a small molecule inhibitor of the hedgehog signaling pathway: effects on basal cell carcinoma-like lesions. Proc Natl Acad Sci USA 100: 4616-4621, 2003.

56. Safholm A, Leandersson K, Dejmek J, et al: A formylated hexapeptide ligand mimics the ability of Wnt5a to impair migration of human breast epithelial cells. J Biol Chem 281 : 2740-2749, 2006.

57. Li S, Christensen C, Kiselyov VV, et al: FGF-derived peptides: functional agonists of FGF receptor. J Neurochem 104: 667-682, 2008

58. Katoh $\mathrm{M}$ and Katoh M: Comparative genomics on RORI and ROR2 orthologs. Oncol Rep 14: 1381-1384, 2005.

59. Fukuda T, Chen L, Endo T, et al: Antisera induced by infusions of autologous Ad-CD154-leukemia B cells identify ROR1 as an oncofetal antigen and receptor for Wnt5a. Proc Natl Acad Sci USA 105: 3047-3052, 2008.

60. Katoh M and Katoh M: STAT3-induced WNT5A signaling loop in embryonic stem cells, adult normal tissues, chronic persistent inflammation, rheumatoid arthritis and cancer. Int J Mol Med 19: 273-278, 2007.

61. Dissanayake SK, Wade M, Johnson CE, et al: Wnt5a/PKC pathway mediates motility in melanoma cells via inhibition of metastasis suppressors and initiation of EMT. J Biol Chem 282: 17259-17271, 2007. 